Bull. Mater. Sci., Vol. 20, No. 6, September 1997, pp. 755-767. (C) Printed in India.

\title{
Computer simulation of surface diffusion of copper, silver and gold
}

\author{
MASAO DOYAMA \\ Teikyo University of Science and Technology, Uenohara, Yamanashi 409-0I, Japan

\begin{abstract}
The binding energies to copper, silver and gold (111) surfaces of self-atom clusters have been calculated. The activation energies of motion of these ad-atom clusters, vacancies and divacancies on copper, silver and gold (111) surface, and of the conversion of ad-atom clusters on (111) and (100) have been calculated by use of $n$-boxly embedded atom potentials and molecular dynanics.
\end{abstract}

Keywords. Simulations; surface diffusion; copper; silver; gold; ad-atoms; ad-atom clusters.

\section{Introduction}

Surface phenomena are becoming important in view of their technological applications as well as in basic sciences. The crystal growth of surface by expitaxial growth is widely used by electronic industries. Surface diffusion is very important for the expitaxial growth. In this paper the binding energies for ad-atom clusters to (111) surface, and the activation energies of the motion and conversion were calculated using molecular dynamics and $n$-body embedded atom method.

\section{Potential}

In metals, the conduction electrons travel from one atom to another atom, and the interaction cannot be represented by a pairwise potential but many-body potentials. The interaction between the $i$ th atom and the $j$ th atom depends not only on the distance between them but also other factors. By the embedded function, surface problems can be treated.

\subsection{Oh and Johnson's potential}

The $n$-body embedded function proposed by Oh and Johnson $(1988,1989)$ is used in this paper. The total energy is given as

$$
\begin{aligned}
E_{\text {total }} & =\Sigma E_{i}, \\
r_{i j} & =\left|r_{i}-r_{j}\right|, \\
E_{i} & =F\left(\rho_{i}\right)+(1 / 2) \Sigma \Phi\left(r_{i j}\right), \\
F(\rho) & =a\left(\rho / \rho_{e}\right)^{n}+b\left(\rho / \rho_{e}\right),
\end{aligned}
$$




$$
\rho_{i}=\Sigma f\left(r_{i j}\right)
$$

Here $E_{\text {total }}$ is the total internal energy, $E_{i}$ the internal energy associated with atom $i, \rho_{i}$ the electron density at atom $i$ due to all other atoms, $F\left(\rho_{i}\right)$ the embedding energy of atom into electron density $\rho_{i}, \Phi\left(r_{i j}\right)$ is the two body central potential between atoms $i$ and $j$ separated by $r_{i j}$, and $f\left(r_{i j}\right)$ the contribution to the electron density at atom $i$ due to atom $j$ at the distance $r_{i j}$ from atom $i$. Further,

$$
\begin{gathered}
f(r)=f_{\text {old }}(r)-f_{\mathrm{c}}(r), \\
f_{\mathrm{old}}=f_{e} \exp \left\{-\beta\left(r / r_{e}\right)-1\right\}, \\
f_{c}(r)=f_{\mathrm{old}}\left(r_{c}\right)+g(r) f_{\mathrm{old}}^{\prime}\left(r_{i}\right) / g^{\prime}(r), \\
\Phi(r)=\Phi_{\mathrm{old}}(r)-\Phi_{c}(r), \\
\Phi_{\mathrm{old}}(r)=\Phi_{e} \exp \left\{-\gamma\left(r / r_{e}-1\right)\right\}, \\
\Phi_{c}(r)=\Phi_{\mathrm{old}}\left(r_{c}\right)+g(r) \Phi_{\mathrm{old}}^{\prime}\left(r_{c}\right) / g^{\prime}\left(r_{c}\right), \\
g(r)=1-\exp \left\{\delta\left(r / r_{e}-r_{c} / r_{e}\right)\right\} .
\end{gathered}
$$

For copper, $\mathrm{Oh}$ and Johnson (1988) give $\beta=5, \gamma=8.5, \delta=20, r_{c}=1.9 r_{e}$, $\Phi_{e}=0.36952 \mathrm{eV}, a=-4.0956, b=-1.6979, n=0.44217$, and $\rho_{e}=12.793$. For silver, $\beta=6, \quad \gamma=7.5, \quad \delta=20, \quad r_{c}=1.9 r_{e}, \quad \Phi_{e}=0.85184 \mathrm{eV}, \quad a=-5.0929, \quad b=-2.9961$, $n=0.65777$, and $\rho_{e}=12.551$. For gold, $\beta=6, \gamma=8, \delta=20, r_{c}=1.9 r_{e}, \Phi_{e}=0.54608 \mathrm{eV}$, $a=-39.701, b=32.428, n=0.92504$, and $\rho_{e}=12.551$.

\subsection{Doyama and Kogure potential}

The $n$-body embedded function proposed by Doyama and Kogure (to be published) was also used in this paper. The total energy is given by

$$
\begin{aligned}
E_{\text {total }} & =\Sigma E_{i,} \\
r_{i j} & =\left|r_{i}-r_{j}\right|, \\
E_{i} & =F\left(\rho_{i}\right)+(1 / 2) \Sigma \Phi\left(r_{i j}\right), \\
F(\rho) & =D \rho_{i} \log \left(\rho_{i}\right), \\
\rho_{i} & =\Sigma f\left(r_{i j}\right), \\
\Phi\left(r_{i j}\right) & =A_{1}\left(r_{c_{1}}-r_{i j}\right)^{2} \exp \left(-c_{1} r_{i j}\right), \\
f\left(r_{i j}\right) & =A_{2}\left(r_{c_{2}}-r_{i j}\right)^{2} \exp \left(-c_{2} r_{i j}\right) .
\end{aligned}
$$


Values of parameters $A_{1}, A_{2}, C_{1}, C_{2}$ and $D$ are given in table 1 . In table 2, experimental and calculated values are listed.

\section{Binding energies of atom clusters to (111) plane}

\subsection{Oh and Johnson's potential}

The binding energies of atom clusters to the (111) surface for copper, silver and gold have been calculated. A crystal used has (111) surface, contains 2310 atoms, 11 atoms in $\langle 110\rangle$ direction, 14 atoms in $\langle 112\rangle$ direction and 15 atoms in $\langle 111\rangle$ direction. 506 or 420 atoms near the centre of (111) surface were relaxed. Let the binding energy of an $N$ ad-atom cluster be $B_{N}$. Each ad-atom has three bonds $\left(3 E_{1}\right)$ to the (111) plane. Let the binding energy per bond in the ad-atom cluster be $E_{2}$ and the number of bonds $m$. The results are shown in tables 3-5 for copper, silver and gold, respectively. For copper, the binding energy of the first ad-atom to (111) plane $(2.60 \mathrm{eV})$ is lower than that of the atom sticking to the next to the atom adsorbed $(3.02 \mathrm{eV})$. The binding energy of the last atom to complete a hexagon

Table 1. Determined potential parameters.

\begin{tabular}{lccc}
\hline & $\mathrm{Cu}$ & $\mathrm{Ag}$ & $\mathrm{Au}$ \\
\hline $\mathrm{A}_{1}$ & $8.28945997705 \times 10^{3}$ & $9.10810768184 \times 10^{3}$ & $5.18021878920 \times 10^{5}$ \\
$\mathrm{~A}_{2}$ & $1.83251035107 \times 10^{-2}$ & $5.25514178418 \times 10^{-2}$ & $1.83065797788 \times 10^{-2}$ \\
$\mathrm{C}_{1}$ & 10.72729128641 & 10.817215101079 & 15.57704835828 \\
$\mathrm{C}_{2}$ & $3.19759369823 \times 10^{-1}$ & 1.38555356572 & $1.28671308081 \times 10^{-5}$ \\
$\mathrm{D}$ & 13.07921251628 & 11.28152486706 & 11.93177438785 \\
\hline
\end{tabular}

Table 2. Experimental values used to fit potential parameters and calculated values.

\begin{tabular}{|c|c|c|c|c|c|}
\hline & & & $\mathrm{Cu}$ & $\mathrm{Ag}$ & $\mathrm{Au}$ \\
\hline \multirow[t]{2}{*}{$C_{11}$} & (cal) & {$\left[10^{12} \mathrm{dyne} / \mathrm{cm}^{2}\right]$} & 1.806 & 1.22 & 1.37 \\
\hline & $(\exp )$ & & 1.684 & $1 \cdot 24$ & $1 \cdot 86$ \\
\hline \multirow[t]{2}{*}{$\mathrm{C}_{12}$} & (cal) & {$\left[10^{12}\right.$ dyne $\left./ \mathrm{cm}^{2}\right]$} & 1.201 & 0.837 & 0.913 \\
\hline & $(\exp )$ & & 1.214 & 0.934 & 1.57 \\
\hline \multirow[t]{2}{*}{$\mathrm{C}_{44}$} & (cal) & {$\left[10^{12}\right.$ dyne $\left./ \mathrm{cm}^{2}\right]$} & 0.710 & 0.489 & 0.492 \\
\hline & $(\exp )$ & & 0.754 & 0.461 & 0.42 \\
\hline \multirow[t]{2}{*}{$\mathrm{E}_{\mathrm{c}}$} & (cal) & {$[\mathrm{eV}]$} & 3.54 & $2 \cdot 85$ & 3.93 \\
\hline & $(\exp )$ & & 3.54 & $2 \cdot 85$ & 3.93 \\
\hline \multirow[t]{2}{*}{$E_{v}^{F}$} & (cal) & {$[\mathrm{eV}]$} & $1 \cdot 3$ & $1 \cdot 10$ & 1.01 \\
\hline & $(\exp )$ & & $1 \cdot 3$ & $1 \cdot 1$ & 0.96 \\
\hline \multirow[t]{2}{*}{$\mathrm{E}_{\mathrm{s}}$} & (cal) & {$\left[\mathrm{ergs} / \mathrm{cm}^{2}\right]$} & 73 & 17 & $52 \cdot 3$ \\
\hline & $(\exp )$ & & 73 & 17 & 55 \\
\hline $\mathbf{a}$ & $(\exp )$ & {$[\mathrm{A}]$} & 3.61496 & 4.0862 & $4 \cdot 07864$ \\
\hline Q & & & $8.78 \times 10^{-3}$ & $1.49 \times 10^{-2}$ & $3 \times 10^{-1}$ \\
\hline
\end{tabular}


Table 3. Binding energies of ad-atom clusters to copper (111) surface using Oh and Johnson potential.

\begin{tabular}{|c|c|c|c|c|c|}
\hline $\begin{array}{l}\text { Number of } \\
\text { atoms in } \\
\text { cluster } N\end{array}$ & Configuration & $\begin{array}{c}\text { Total binding } \\
\text { energy } \\
B=3 n_{1} E_{1} \\
+n_{2} E_{2}(\mathrm{eV})\end{array}$ & $\begin{array}{c}\text { Number of } \\
\text { nearest } \\
\text { neighbours } \\
3 n_{1}+n_{2}\end{array}$ & $\begin{array}{l}\text { Energy per } \\
\text { bond } \\
B /\left(3 n_{1}+n_{2}\right)\end{array}$ & $\begin{array}{l}\text { Energy per } \\
\text { bond within } \\
\text { clusters } B_{2}\end{array}$ \\
\hline 1 & $\bullet$ & $2 \cdot 60$ & $3(3 \times 1)$ & 0.87 & \\
\hline 2 & $\bullet$ & 5.62 & $7(=3 \times 2+1)$ & 0.80 & 0.42 \\
\hline 3 & $\therefore$ & 8.97 & $12(=3 \times 3+3)$ & 0.76 & 0.39 \\
\hline 4 & $\because$ & 12.28 & $17(=4 \times 3+5)$ & 0.72 & 0.38 \\
\hline 4 & & 11.94 & $16(=4 \times 3+4)$ & 0.75 & 0.39 \\
\hline 5 & & 15.57 & $22(=5 \times 3+7)$ & 0.71 & 0.37 \\
\hline 6 & & 18.82 & $27(=6 \times 3+9)$ & 0.70 & 0.36 \\
\hline 6 & $\bullet$ & $18 \cdot 47$ & $25(=6 \times 3+7)$ & 0.74 & 0.36 \\
\hline 6 & $\bullet$ & 18.85 & $27(=6 \times 3+9)$ & 0.70 & 0.36 \\
\hline 7 & $\because$ & 22.37 & $33(=7 \times 3+12)$ & 0.68 & 0.35 \\
\hline 7 & & 21.76 & $32(=7 \times 3+11)$ & 0.68 & 0.32 \\
\hline
\end{tabular}

with one atom at the centre (7-atom cluster) $(3.55 \mathrm{eV})$ is higher than other cases. The difference between the binding energy of an atom at a normal site $(N$-site: form fcc structure) and that of an atom at hexagonal site ( $H$-site) which forms a stacking fault was calculated to be $0.004 \mathrm{eV}$. For silver, the binding energy of the first atom to (111) plane $(2 \cdot 13 \mathrm{eV})$ is lower than that of the atom sticking to the next to the atom adsorbed $(2.38 \mathrm{eV})$. The binding energy of the last atom to complete a hexagon with one atom at the centre (7-atom cluster) $(2.9 \mathrm{eV})$ is higher than other cases. The difference between the binding energy of an atom at a normal site (form fcc structure) and that of an atom at a $H$-site which forms a stacking fault was calculated to be $0.0085 \mathrm{eV}$. For gold the binding energy of the first atom to $(111)$ plane $(2.90 \mathrm{eV})$ is lower than that of the atom sticking to the next to the atom adsorbed $(3.42 \mathrm{eV})$. The binding energy of the last atom to complete a hexagon with one atom at the centre (7-atom cluster) $(4.04 \mathrm{eV})$ is higher than other cases. The difference between the binding energy of an atom at a normal site (form fcc structure) and that of an atom at a $H$-site which forms a stacking fault was calculated to be $0.0028 \mathrm{eV}$.

\subsection{Doyama and Kogure potential}

The binding energies of atom clusters to the (111) surface for copper, silver and 
Table 4. Binding energies of ad-atom clusters to silver (111) surface using Oh and Johnson potential.

\begin{tabular}{|c|c|c|c|c|c|}
\hline $\begin{array}{l}\text { Number of } \\
\text { atoms in } \\
\text { cluster } N\end{array}$ & Configuration & $\begin{array}{l}\text { Total binding } \\
\text { energy } \\
B=3 n_{1} E_{1} \\
+n_{2} E_{2}(\mathrm{eV})\end{array}$ & $\begin{array}{c}\text { Number of } \\
\text { nearest } \\
\text { neighbours } \\
3 n_{1}+n_{2}\end{array}$ & $\begin{array}{l}\text { Energy per } \\
\text { bond } \\
B /\left(3 n_{1}+n_{2}\right)\end{array}$ & $\begin{array}{l}\text { Energy per } \\
\text { bond within } \\
\text { clusters. } B_{2}\end{array}$ \\
\hline 1 & & $2 \cdot 13$ & $3(=3 \times 1)$ & 0.71 & \\
\hline 2 & & 4.51 & $7(=3 \times 2+1)$ & 0.64 & 0.25 \\
\hline 3 & & $7 \cdot 19$ & $12(=3 \times 3+3)$ & $0 \cdot 60$ & 0.24 \\
\hline 3 & - & 6.85 & $11(=3 \times 3+2)$ & 0.62 & 0.24 \\
\hline 3 & & 685 & $11(=3 \times 3+2)$ & 0.62 & $0 \cdot 24$ \\
\hline 4 & & 9.82 & $17(=3 \times 4+5)$ & 0.58 & 0.26 \\
\hline 4 & & 9.51 & $16(=3 \times 4+4)$ & 0.59 & 0.25 \\
\hline 4 & & $9 \cdot 20$ & $15(=3 \times 4+3)$ & 0.61 & $0 \cdot 23$ \\
\hline 4 & & 9.20 & $15(=3 \times 4+3)$ & 0.61 & 0.23 \\
\hline 5 & & $12 \cdot 44$ & $22(=3 \times 5+7)$ & 0.57 & 0.26 \\
\hline 5 & & 11.87 & $20(=3 \times 5+5)$ & 0.59 & $0 \cdot 25$ \\
\hline 5 & & 11.56 & $19(=3 \times 5+4)$ & 0.61 & 0.23 \\
\hline 5 & $\because$ & 11.56 & $19(=3 \times 4+4)$ & 0.61 & 0.23 \\
\hline 6 & & $15 \cdot 04$ & $27(=3 \times 6+9)$ & 0.56 & $0 \cdot 25$ \\
\hline 6 & & $15 \cdot 04$ & $27(=3 \times 6+9)$ & 0.56 & 0.26 \\
\hline 6 & & $15 \cdot 04$ & $27(=3 \times 6+9)$ & 0.56 & 0.26 \\
\hline 7 & $\because$ & 17.94 & $33(=3 \times 7+12)$ & 0.54 & 0.26 \\
\hline
\end{tabular}

gold using Doyama and Kogure potential have been calculated for the same crystal structure as the one used in the preceding subsection. The results are shown in tables 6 and 7 for copper and silver respectively. For copper the binding energy of the first ad-atom to (111) plane $(2.42 \mathrm{eV})$ is lower than that of the atom sticking to the next to the atom adsorbed $(2.92 \mathrm{eV})$. The difference between the binding energy of an atom at a normal site (form fcc structure) and that of an atom at a $H$-site which forms a stacking fault was calculated to be $0.013 \mathrm{eV}$. For silver the binding energy of the first atom to $(111)$ plane $(1.87 \mathrm{eV})$ is lower than that of the atom sticking to the next to the atom adsorbed $(2.3 \mathrm{eV})$. The difference between the binding energy of an atom at a normal site (form fcc structure) and that of an atom at a $H$-site which forms a stacking fault was calculated to be $0.0041 \mathrm{eV}$. 


\section{Motion of an ad-atom on (111) and (100) (figure 1)}

\subsection{Oh and Johnson's potential}

For copper the binding energy of an atom on (111) surface at the $N$-site and at the $H$-site was calculated to be $2.61 \mathrm{eV}$ and $2.60 \mathrm{eV}$, respectively. The activation energy for the motion of ad-atom from $N$-site to $H$-site, $E_{1 A}^{M}(N \rightarrow H)$ (111), was calculated to be $0.043 \mathrm{eV}$ with relaxation of 507 atoms around and including the ad-atom. The activation energy for the motion of ad-atom from $H$-site to $N$-site, $E_{1 A}^{M}(H \rightarrow N)(111)$, was $0.039 \mathrm{eV}$ which are quite small. The energy difference between $N$-site and $H$-site was $0.004 \mathrm{eV}$.

For silver the binding energy of an atom on (111) surface at the $N$ - and $H$-sites was calculated to be $2.126 \mathrm{eV}$ and $2.118 \mathrm{eV}$, respectively. The activation energy for the motion of ad-atom from $N$-site to $H$-site, $E_{1 A}^{M}(N \rightarrow H)$ (111), was calculated

Table 5. Binding energies of ad-atom clusters to gold (111) surface using Oh and Johnson potential.

\begin{tabular}{|c|c|c|c|c|c|}
\hline $\begin{array}{l}\text { Number of } \\
\text { atoms in } \\
\text { cluster } \mathrm{N}\end{array}$ & Configuration & $\begin{array}{l}\text { Total binding } \\
\text { energy } \\
B=3 n_{1} E_{1} \\
+n_{2} E_{2} \cdot(\mathrm{eV})\end{array}$ & $\begin{array}{c}\text { Number of } \\
\text { nearest } \\
\text { neighbours } \\
3 n_{1}+n_{2}\end{array}$ & $\begin{array}{l}\text { Energy per } \\
\text { bond } \\
B /\left(3 n_{1}+n_{2}\right)\end{array}$ & $\begin{array}{l}\text { Energy per } \\
\text { bond within } \\
\text { clusters } B_{2}\end{array}$ \\
\hline 1 & & 2.91 & $3(=3 \times 1)$ & 0.97 & \\
\hline 2 & & 6.32 & $7(=3 \times 2+1)$ & 0.90 & 0.50 \\
\hline 3 & & $10 \cdot 16$ & $12(=3 \times 3+3)$ & 0.85 & 0.48 \\
\hline 3 & & 9.62 & $11(=3 \times 3+2)$ & 0.87 & 0.45 \\
\hline 3 & & 9.60 & $11(=3 \times 3+2)$ & 0.87 & 0.44 \\
\hline 4 & & 13.83 & $17(=3 \times 4+5)$ & 0.81 & 0.44 \\
\hline 4 & & 13.41 & $16(=3 \times 4+4)$ & 0.84 & 0.44 \\
\hline 4 & & 12.94 & $15(=3 \times 4+3)$ & 0.86 & 0.43 \\
\hline 4 & & 12.90 & $15(=3 \times 4+3)$ & 0.86 & 0.42 \\
\hline 5 & & 17.51 & $22(=3 \times 5+7)$ & 0.80 & 0.42 \\
\hline 5 & & $16 \cdot 75$ & $20(=3 \times 5+5)$ & 0.84 & 0.44 \\
\hline 5 & & 16.27 & $19(=3 \times 5+4)$ & 0.86 & 0.43 \\
\hline 5 & & 16.24 & $19(=3 \times 5+4)$ & 0.85 & 0.42 \\
\hline 6 & & $21 \cdot 12$ & $27(=3 \times 6+9)$ & 0.78 & 0.41 \\
\hline 6 & & $21 \cdot 18$ & $27(=3 \times 6+9)$ & 0.78 & 0.40 \\
\hline 7 & & $25 \cdot 16$ & $33(=3 \times 7+12)$ & 0.64 & 0.40 \\
\hline
\end{tabular}


to be $0.0559 \mathrm{eV}$ with relaxation of 507 atoms around and including the ad-atom. The activation energy for the motion of ad-atom from $H$-site to $N$-site $E_{1 A}^{M}(H \rightarrow N)(111)$, was $0.0464 \mathrm{eV}$. The activation energies are quite small. The energy difference between $N$ - and $H$-sites was $0.0085 \mathrm{eV}$.

For gold the binding energy of an atom on (111) surface at the $\mathrm{N}$ - and $\mathrm{H}$-sites was calculated to be $2.91 \mathrm{eV}$ and $2.90 \mathrm{eV}$ respectively. The activation energy for the motion of ad-atom from $N$-site to $H$-site, $E_{1 A}^{M}(N \rightarrow H)(111)$, was calculated to be $0.084 \mathrm{eV}$ with relaxation of 507 atoms around and including the ad-atom. The activation energy for the motion of ad-atom from $H$-site to $N$-site, $E_{1 A}^{M}(H \rightarrow N)$ (111), was $0.074 \mathrm{eV}$. The activation energies are quite small. The energy difference between $N$-site and $H$-site was $0.010 \mathrm{eV}$.

The activation energy for the motion of an ad-atom on (100), $E_{1 A}^{M}(100)$, were calculated to be 0.50 and $0.81 \mathrm{eV}$ for copper and gold, which are much higher than that on (111). (111) plane has the highest atomic density and, is smooth, but

Table 6. Binding energies of ad-atom clusters to copper (111) surface using Doyama and Kogure potential.

\begin{tabular}{|c|c|c|c|c|c|}
\hline $\begin{array}{l}\text { Number of } \\
\text { atoms in } \\
\text { cluster N }\end{array}$ & Configuration & $\begin{array}{l}\text { Total binding } \\
\text { energy } \\
B=3 n_{1} E_{1} \\
+n_{2} E_{2}(\mathrm{eV})\end{array}$ & $\begin{array}{c}\text { Number of } \\
\text { nearest } \\
\text { neighbours } \\
3 n_{1}+n_{2}\end{array}$ & $\begin{array}{l}\text { Energy per } \\
\text { bond } \\
B /\left(3 n_{1}+n_{2}\right)\end{array}$ & $\begin{array}{l}\text { Energy per } \\
\text { bond within } \\
\text { clusters } B_{2}\end{array}$ \\
\hline 1 & & $2 \cdot 424$ & 3 & 0.81 & \\
\hline 2 & & $5 \cdot 336$ & $7(=3 \times 2+1)$ & 0.76 & 0.49 \\
\hline 2 & $\bullet(110)$ & $5 \cdot 314$ & $7(=3 \times 2+1)$ & 0.76 & 0.47 \\
\hline 3 & & $8 \cdot 648$ & $12(=3 \times 3+3)$ & 0.72 & 0.46 \\
\hline 4 & & 11.420 & $16(=3 \times 4+4)$ & 0.71 & 0.43 \\
\hline 4 & & $11 \cdot 116$ & $15(=3 \times 4+3)$ & 0.74 & 0.47 \\
\hline 4 & & 11.097 & $15(=3 \times 4+3)$ & 0.76 & 0.47 \\
\hline 5 & & $15 \cdot 176$ & $22(=3 \times 5+7)$ & 0.69 & 0.44 \\
\hline 5 & & 14.009 & $19(=3 \times 5+4)$ & 0.74 & 0.47 \\
\hline 5 & & 13.990 & $19(=3 \times 5+4)$ & 0.74 & 0.47 \\
\hline 5 & & 14.415 & $20(=3 \times 5+5)$ & 0.72 & 0.46 \\
\hline 5 & $\bullet \bullet$ & 13.914 & $19(=3 \times 5+4)$ & 0.73 & 0.45 \\
\hline 6 & & 18.401 & $27(=3 \times 6+9)$ & 0.68 & 0.43 \\
\hline 6 & & $18 \cdot 425$ & $27(=3 \times 6+9)$ & 0.68 & 0.43 \\
\hline 6 & & 17.679 & $25(=3 \times 6+7)$ & 0.71 & 0.45 \\
\hline 6 & & $16 \cdot 292$ & $24(=3 \times 6+5)$ & & \\
\hline
\end{tabular}


(100) plane does not have the highest atomic density. The surface is not very smooth. Therefore, it is reasonable that the activation energy for the motion of a single ad-atom on (100) is higher than that on (111).

\subsection{Doyama and Kogure potential}

For copper the binding energy of an atom on (111) surface at the $\mathrm{N}$ - and $\mathrm{H}$-sites was calculated to be $2.61 \mathrm{eV}$ and $2.60 \mathrm{eV}$ respectively. The activation energy for the motion of ad-atom from $N$-site to $H$-site, $E_{1 A}^{M}(N \rightarrow H)(111)$, was calculated to be $0.055 \mathrm{eV}$ with relaxation of 507 atoms around and including the ad-atom. The activation energy for the motion of ad-atom from $H$-site to $N$-site, $E_{1 A}^{M}(H \rightarrow N)$ (111), was $0.042 \mathrm{eV}$. These are quite small. The energy difference between $N$-site and $H$-site was $0.013 \mathrm{eV}$.

For silver the binding energy of an atom on (111) surface at the $N$-site and at

Table 7. Binding energies of ad-atom clusters to silver (111) surface using Doyama and Kogure potential.

\begin{tabular}{|c|c|c|c|c|c|}
\hline $\begin{array}{l}\text { Number of } \\
\text { atoms in } \\
\text { cluster } N\end{array}$ & Configuration & $\begin{array}{l}\text { Total binding } \\
\text { energy } \\
B=3 n_{1} E_{1} \\
+n_{2} E_{2}(\mathrm{eV})\end{array}$ & $\begin{array}{c}\text { Number of } \\
\text { nearest } \\
\text { neighbours } \\
3 n_{1}+n_{2}\end{array}$ & $\begin{array}{l}\text { Energy per } \\
\text { bond } \\
B /\left(3 n_{1}+n_{2}\right)\end{array}$ & $\begin{array}{l}\text { Energy per } \\
\text { bond within } \\
\text { clusters } B_{2}\end{array}$ \\
\hline 1 & & 1.867 & 3 & 0.62 & \\
\hline 2 & & $4 \cdot 174$ & $7(=3 \times 2+1)$ & 0.60 & 0.44 \\
\hline 3 & & 6.837 & $12(=3 \times 3+3)$ & 0.57 & 0.41 \\
\hline 3 & & 6.460 & $11(=3 \times 3+2)$ & 0.59 & 0.43 \\
\hline 3 & & $6 \cdot 452$ & $11(=3 \times 3+2)$ & 0.59 & 0.43 \\
\hline 4 & & 9.459 & $17(=3 \times 4+5)$ & 0.56 & 0.40 \\
\hline 4 & & 9.097 & $16(=3 \times 4+4)$ & 0.57 & 0.41 \\
\hline 4 & & $8 \cdot 737$ & $15(=3 \times 4+3)$ & 0.58 & 0.42 \\
\hline 4 & & 8.730 & $15(=3 \times 4+3)$ & 0.58 & 0.42 \\
\hline 5 & & 11.381 & $20(=3 \times 5+5)$ & 0.57 & 0.41 \\
\hline 5 & & 11.020 & $19(=3 \times 5+4)$ & 0.58 & 0.42 \\
\hline 5 & & $11 \cdot 012$ & $19(=3 \times 5+4)$ & 0.58 & 0.42 \\
\hline 5 & & 10.973 & $19(=3 \times 5+4)$ & 0.58 & 0.41 \\
\hline 6 & & 14.667 & $27(=3 \times 6+9)$ & 0.54 & $0 \cdot 39$ \\
\hline 6 & & 14.658 & $27(=3 \times 6+9)$ & 0.54 & $0 \cdot 38$ \\
\hline 6 & & 14.647 & $27(=3 \times 6+9)$ & 0.54 & 0.38 \\
\hline
\end{tabular}




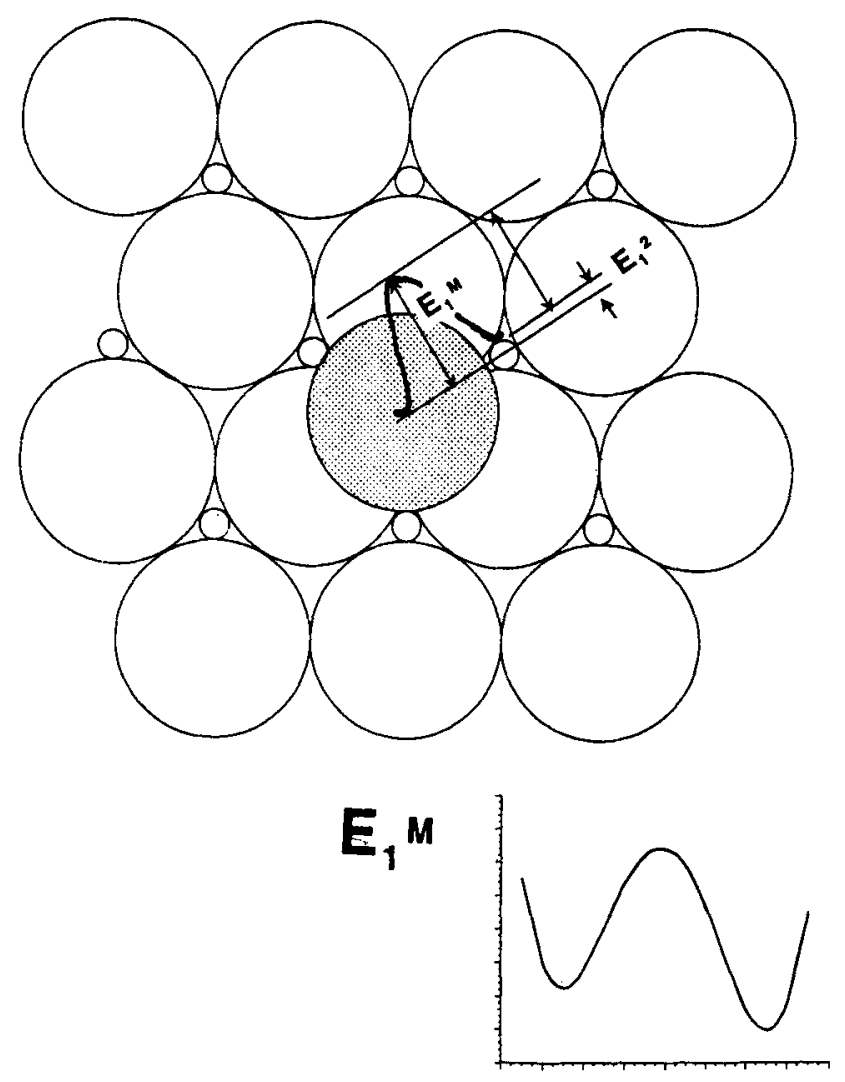

Figure 1. Motion of an ad-atom on (1l!). From $N$-site to $H$-site and from $H$-site to $N$-sitc.

the $H$-site was calculated to be $1.867 \mathrm{eV}$ and $1.864 \mathrm{eV}$. The activation energy for the motion of ad-atom from $N$-site to $H$-site, $E_{1 A}^{M}(N \rightarrow H)$ (111), was calculated to be $0.058 \mathrm{eV}$ with relaxation of 507 atoms around and including the ad-atom. The activation energy for the motion of ad-atom from $H$-site to $N$-site, $E_{1 \lambda}^{M}(H \rightarrow N)$ (111), was $0.054 \mathrm{eV}$. The activation energies are quite small. The energy difference between $\mathrm{N}$-site and $\mathrm{H}$-site was $0.004 \mathrm{eV}$.

\section{Di ad-atoms on (111) and (100) (figure 2)}

\subsection{Oh and Johnson's potential}

For copper the binding energy of a di ad-atom to (111) plane was calculated to be $5.62 \mathrm{eV}$ (table 3). The binding energy of the second atoms was $3.02 \mathrm{eV}$ which is higher than the first ad-atom to the (111) surface $(2.60 \mathrm{eV})$. The activation energy for the motion of a di ad-atom on (111), $E_{2 A}^{M}$ (111), was calculated to be $0.30 \mathrm{eV}$ with relaxation of 420 atoms around and including the ad-atoms. A di ad-atom moves by butterfly motion, without breaking the bond between two ad-atoms. 


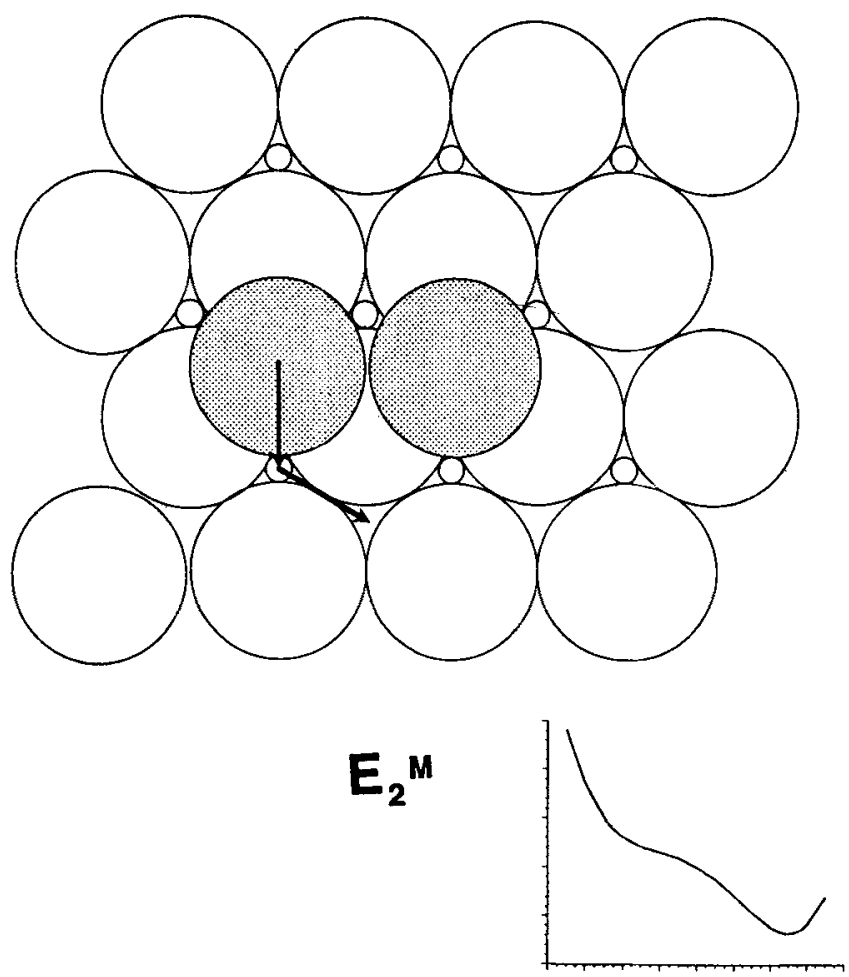

Figure 2. Motion of a di ad-atom. The di ad-atom has to be partially broken while it moves.

The activation energy for the motion of a di ad-atom is much higher than that of a single ad-atom. An atom (atom 1) of a di ad-atom was moved away from the other atom (atom 2).

For silver the binding energy of a di ad-atom to (111) plane was calculated to be $4.51 \mathrm{eV}$ (table 4). The binding energy of the second atoms was $2.38 \mathrm{eV}$ which is higher than the first ad-atom to the (111) surface $(2 \cdot 1 \mathrm{eV})$. The activation energy for the motion of di ad-atoms on (111) was calculated to be $0.148 \mathrm{eV}$ with relaxation of 420 atoms around and including the ad-atoms. The activation energy for the motion of di ad-atoms is much higher than that of a single ad-atom. An atom (atom 1) of di ad-atoms was moved away from the other atom (atom 2).

For gold the binding energy of a di ad-atom to (111) plane was calculated to be $6.32 \mathrm{eV}$ (table 5). The binding energy of the second atoms was $3.42 \mathrm{eV}$ which is higher than the first ad-atom to the (111) surface $(2.90 \mathrm{eV})$. The activation energy for the motion of a di ad-atom on (111), $E_{2 A}^{M}$ (111), was calculated to be $0.14 \mathrm{eV}$ with relaxation of 420 atoms around and including the ad-atoms. A di ad-atom moves by butterfly motion, without breaking the bond between two ad-atoms. The activation energy for the motion of a di ad-atom is much higher than that of a single ad-atom. An atom (atom 1) of a di ad-atom was moved away from the other atom (atom 2).

For the motion of a di ad-atom on (100), one of the di ad-atom of the di ad-atom in the nearest neighbour distance has to be broken first into the next 
nearest neighbour position and the other ad-atom moves. For copper the activation energy for the conversion, $E_{2 A}^{M}(\mathrm{nn} \rightarrow \mathrm{nnn})$ was found to be $0.60 \mathrm{eV}$ and $E_{2 A}^{M}(\mathrm{nnn} \rightarrow \mathrm{nn})$ was $0.30 \mathrm{eV}$. Therefore, the activation energy for the motion of di ad-atom on (100) is $0.60 \mathrm{eV}$ for copper. The activation energy for the break up of a di ad-atom from the nearest neighbour to $2 d$ ( $d$ is the nearest neighbour distance) in [110] was found to be $0.90 \mathrm{eV}$ for copper.

\subsection{Doyama and Kogure potential}

For copper the binding energy of a di ad-atom to (111) plane was calculated to be $5.34 \mathrm{eV}$ (table 6). The binding energy of the second atoms was $2.92 \mathrm{eV}$ which is higher than the first ad-atom to the (111) surface $(2.42 \mathrm{eV})$. The activation energy for the motion of a di ad-atom on (111), $E_{2 A}^{M}(111)$, was calculated to be $0.12 \mathrm{eV}$ with relaxation of 420 atoms around and including the ad-atoms. A di ad-atom moves by butterfly motion, without breaking the bond between two ad-atoms. The activation energy for the motion of a di ad-atom is much higher than that of a single ad-atom. An atom (atom 1) of a di ad-atom was moved away from the other atom (atom 2).

For silver the binding energy of a di ad-atom to (111) plane was calculated to be $4.17 \mathrm{eV}$ (table 7). The binding energy of the second atom was $2.30 \mathrm{eV}$ which is higher than the first ad-atom to the (111) surface $(1.87 \mathrm{eV})$.

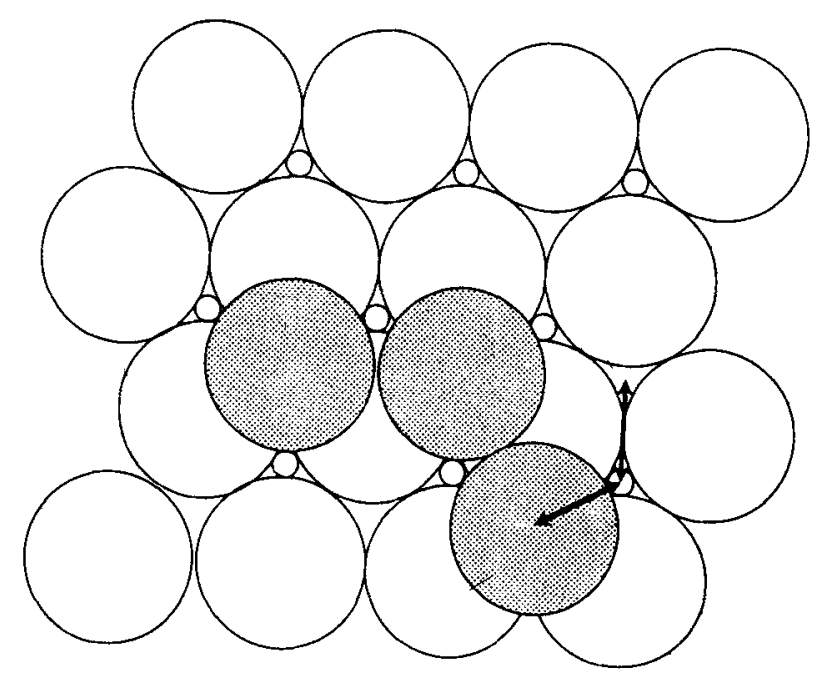

$$
\frac{E_{3}^{180} \rightarrow E_{3}^{120}}{E_{3}^{120} \rightarrow E_{3}^{180}}
$$

Figure 3. Motion of a 3 ad-atom. $E_{3}^{|8| \mid} \rightleftarrows E_{3}^{|2|}$ conversion. 


\section{Motion of 3 ad-atoms on (111) and (100) (figure 3)}

\subsection{Oh and Johnson's potential}

The most stable configuration of a tri ad-atom is triangular for copper, silver and gold. The configurations of the tri ad-atom shown in figure 3 will be called $60^{\circ}$ tri ad-atom, $120^{\circ}$ tri ad-atom and $180^{\circ}$ tri ad-atom, respectively. The activation energies for the transformation from $60^{\circ}$ to $120^{\circ}$ tri ad-atoms, $E_{3 A}^{M}(60 \rightarrow 120)(111)$, was calculated to be $0.413 \mathrm{eV}$ and $0.632 \mathrm{eV}$, for silver and gold, respectively. The activation energies for the transformation from $120^{\circ}$ to $60^{\circ}$ tri ad-atoms, $E_{3 A}^{M}(120 \rightarrow 60)(111)$, were calculated to be $0.083 \mathrm{eV}$ and $0.116 \mathrm{eV}$, for silver and gold, respectively, which are quite low. The activation energies for the transformation from $180^{\circ}$ to $120^{\circ}$ tri ad-atom, $E_{3 A}^{M}(180 \rightarrow 120)(111)$, was calculated to be $0.156 \mathrm{eV}$ and $0.118 \mathrm{eV}$ for silver and gold respectively. Those from $120^{\circ}$ to $180^{\circ}$, $E_{3 A}^{M}(120 \rightarrow 180)(111)$, were $0.140 \mathrm{eV}$.

The activation energy for the conversion of tri ad-atom $90^{\circ}$ to $90^{\circ}$, $E_{34}^{M}(90 \rightarrow 90)(100)$ was found to be $0.43 \mathrm{eV}$ for copper. The activation energy for the conversion of tri ad-atom $90^{\circ}$ to $135^{\circ}, E_{3 A}^{M}(90 \rightarrow 135)(100)$, was found to be $0.67 \mathrm{eV}$ for copper. The activation energy for the conversion of $180^{\circ}$ tri ad-atom to $135^{\circ}, E_{3 A}^{M}(180 \rightarrow 135)(100)$ was found to be $0.60 \mathrm{eV}$ for copper. The energy for a $180^{\circ}$ tri ad-atom to convert into $90^{\circ}$ next neighbours $E_{3 A}^{\mathcal{M}}(180 \rightarrow 90 \mathrm{nnn})(100)$, was found to be $0.70 \mathrm{eV}$ for copper.

\section{A surface vacancy on (111)}

\subsection{Oh and Johnson's potential}

It was found that ad-atoms move quite easily on (111) plane. How does a vacancy on (111) plane move? The formation energy of a surface vacancy is then $1.66 \mathrm{eV}$. The activation energy for the motion of a surface vacancy on (111) was calculated to be $0.958 \mathrm{eV}$. A vacancy on (111) plane is much harder to move compared with an ad-atom. For silver formation energy was found to be $4 \cdot 163 \mathrm{eV}$. Since the binding energy of an ad-atom is $2.60 \mathrm{eV}$, the formation energy is then $1.66 \mathrm{eV}$. The activation energy for the motion of a vacancy on (111) was calculated to be $0.958 \mathrm{eV}$. A vacancy on (111) plane is much harder to move compared with an ad-atom. For gold, formation energy was found to be $4.163 \mathrm{eV}$. Since the binding energy of an ad-atom is $2.60 \mathrm{eV}$, the formation energy is then $1.66 \mathrm{eV}$. The activation energy for the motion of a vacancy on (111) was calculated to be $0.785 \mathrm{eV}$. A vacancy on (111) plane is much harder to move compared with an ad-atom.

\section{A surface divacancy on (111)}

\subsection{Oh and Johnson's potential}

For copper the formation energy of a divacancy on (111) was calculated to be $8.190 \mathrm{eV}$. Since the binding energy of di ad-atoms is $5.62 \mathrm{eV}$, the formation energy is $2.57 \mathrm{eV}$. It is well known that a divacancy is much easier to move compared with a vacancy in fcc metals. How is the motion of divacancy on (111) plane? 
The activation energy for the motion of a surface divacancy on (111) was calculated to be $0.916 \mathrm{eV}$. A divacancy on (111) moves as hard as single vacancy on (111). The saddle point for the motion of divacancy is the centre of the trivacancy.

For gold, the formation energy of a divacancy on (111) was calculated to be $8.190 \mathrm{eV}$. Since the binding energy of di ad-atoms is $5.62 \mathrm{eV}$, the formation energy is $2.57 \mathrm{eV}$. It is well known that a divacancy is much easier to move compared with a vacancy in fcc metals. How is the motion divacancy on (111) plane? The activation energy for the motion of divacancy on (111) was calculated to be $0.916 \mathrm{eV}$. A divacancy on (111) moves as hard as single vacancy on (111). The saddle point for the motion of divacancy is the centre of the trivacancy.

For silver the formation energy of a divacancy on (111) was calculated to be $8.190 \mathrm{eV}$. Since the binding energy of di ad-atoms is $5.62 \mathrm{eV}$, the formation energy is $2.57 \mathrm{eV}$. The activation energy for the motion of divacancy on (111) was calculated to be $0.916 \mathrm{eV}$. A divacancy on (111) moves as hard as single vacancy on (111). The saddle point for the motion of divacancy is the centre of the trivacancy.

\section{Vacancies and divacancies in bulk}

\subsection{Oh and Johnson's potential}

The activation energies for the motion of a vacancy and divacancy was calculated to be $0.79 \mathrm{eV}$ and $0.59 \mathrm{eV}$ for gold. The experimental values are $0.82 \mathrm{eV}$ and $0.66 \mathrm{eV}$, respectively.

\section{Acknowledgements}

The author expresses his gratitude to Professor Saburo Nagakura and Professor P Rama Rao and the Japan Society for Promotion of Science, Japan and the Department of Science and Technology, India for the support of the symposium.

The author acknowledges the assistance of Mr J Takano and M Mochizuki for their calculation of activation energies on (100). This research is supported by the Grant-in-aid by the Ministry of Education, Science and Culture under Priority Area Physics and Chemistry of Graded Functional Materials.

\section{References}

Oh D J and Johnson R A 1988 J. Mater. Res. 3471

Oh D J and Johnson R A 1989 in Atomic simulation of materials (eds) V Vitek and D J Srolovitz (New York: Plenum Press) p. 233 